\title{
Assessment of Undiscovered Conventional Oil and Gas Resources of North Africa, 2012
}

Using a geology-based assessment methodology, the U.S. Geological Survey estimated means of 19 billion barrels of technically recoverable undiscovered conventional oil and 370 trillion cubic feet of undiscovered conventional natural gas resources in 8 geologic provinces of North Africa.

\section{Introduction}

The U.S. Geological Survey (USGS) assessed the potential for undiscovered conventional oil and gas accumulations within priority geologic provinces of North Africa as part of the USGS World Petroleum Resources Project. Eight priority geologic provinces were assessed in this study, which represents a reassessment of North Africa last published in 2000 (U.S. Geological Survey World Energy Assessment Team, 2000). The eight geologic provinces include (1) Nile Delta Basin; (2) Sirte Basin; (3) Pelagian Basin; (4) Trias/Ghadames Basin; (5) Hamra Basin; (6) Illizi Basin; (7) Grand Erg/Ahnet Basin; and (8) Essaouira Basin (fig. 1). Resource estimates for the Nile Delta, Sirte, and Pelagian Basin provinces were published previously (Kirschbaum and others, 2010; Whidden and others, 2011), but are included here for a more complete view of undiscovered conventional oil and gas resources across North Africa.

The assessment methodology included a complete geologic framework description for each province based mainly on published literature and definitions of petroleum systems and assessment units (AU) within these systems. Exploration and discovery history data were a critical part of the methodology used to estimate sizes and numbers of undiscovered conventional accumulations. Each AU was assessed for undiscovered oil and nonassociated gas accumulations, and co-product ratios were used to calculate the volumes of associated gas (gas in oil fields) and volumes of natural gas liquids.

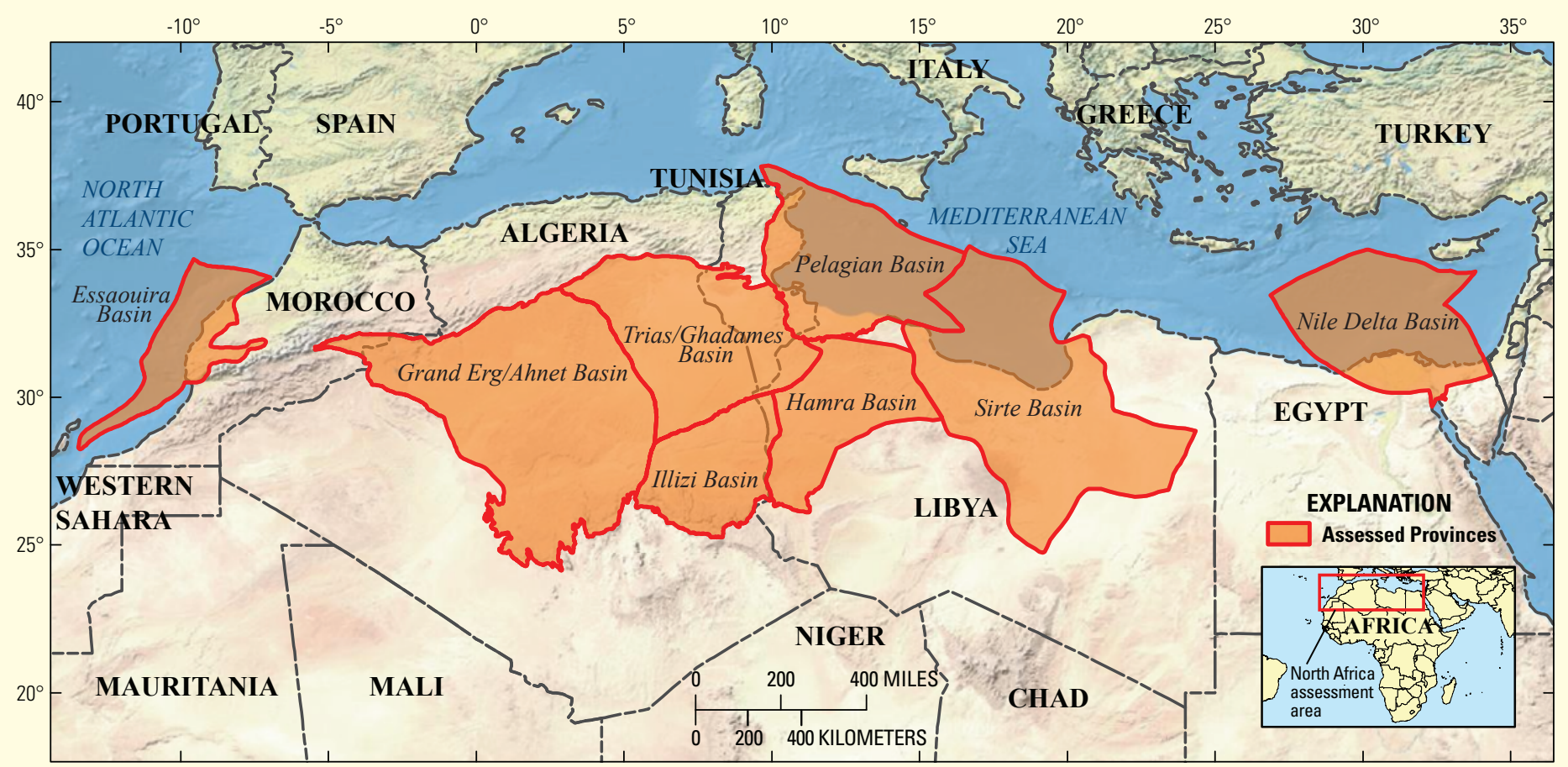

Figure 1. Locations of eight priority geologic provinces of North Africa assessed in this study of undiscovered conventional oil and gas resources. 


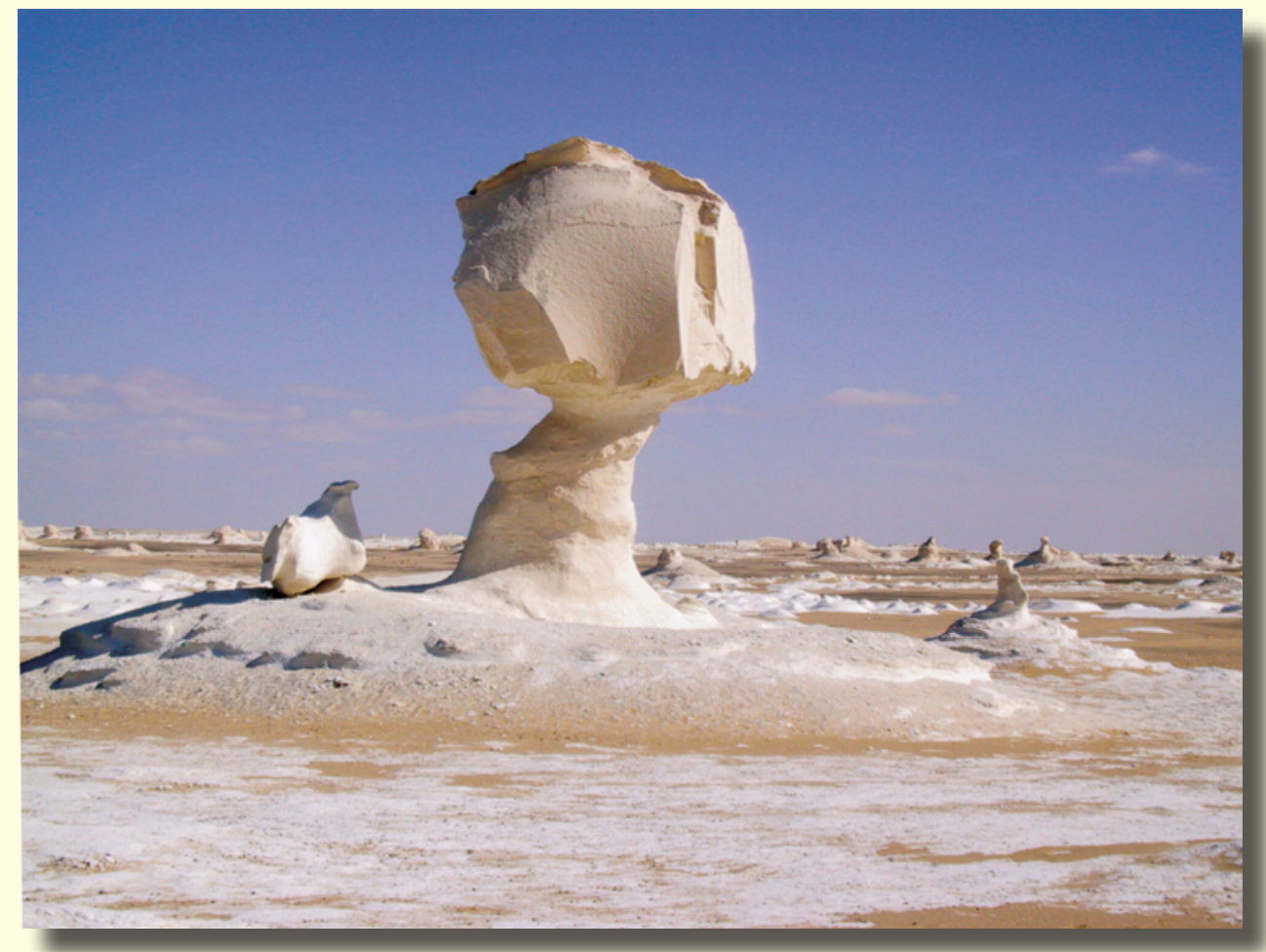

Hoodoos (thin spires of rock) in the Sahara Desert, Egypt. Hoodoos are composed of fine-grained, chalky limestone. Photo by K.J. Whidden, 2005.

\section{Resource Summary}

The USGS assessed undiscovered conventional oil and gas resources in 18 AUs within eight geologic provinces, with the following estimated mean totals: (1) for conventional oil resources, 18,618 million barrels of oil (MMBO), with a range from 6,846 to 37,460 MMBO; (2) for undiscovered conventional gas, 370,375 billion cubic feet of gas (BCFG), with a range from 149,541 to 712,430 $\mathrm{BCFG}$; and (3) for natural gas liquids (NGL), 12,553 MMBNGL, with a range from 4,809 to 24,785 MMBNGL.

Of the mean undiscovered conventional oil resource of $18,618 \mathrm{MMBO}$, about 41 percent $(7,557 \mathrm{MMBO})$ is estimated to be in the Offshore Salt Structures AU, offshore Morocco.
Other significant AUs for potential undiscovered oil include the Offshore Sirte Basin AU (2,267 MMBO), Onshore Sirte Carbonate-Clastic AU (1,278 MMBO), and the Berkine Paleozoic and Mesozoic Reservoirs AU (1,839 MMBO) of the Trias/ Ghadames Basin. Of the mean undiscovered gas resource of $370,375 \mathrm{BCFG}$, about 59 percent $(217,313 \mathrm{BCFG})$ is estimated to be in the Nile Cone AU. Other significant AUs for potential undiscovered gas resources include the Offshore Salt Structures AU of Morocco (45,208 BCFG), the Offshore Sirte Basin AU $(22,637$ BCFG), and the Gourara Paleozoic Reservoirs AU $(15,559$ BCFG) of the Grand Erg/Ahnet Basin Province. These four AUs encompass about 81 percent of the undiscovered gas resource. 
Table 1. Assessment results for undiscovered conventional oil and gas resources for provinces of North Africa.

[MMBO, million barrels of oil; BCFG, billion cubic feet of gas; MMBNGL, million barrels of natural gas liquids. Results shown are fully risked estimates. For gas accumulations, all liquids are included as NGL (natural gas liquids) category. Undiscovered gas resources are the sum of nonassociated (gas in gas fields) and associated gas (gas in oil fields). F95 represents a 95 percent chance of at least the amount tabulated-other fractiles are defined similarly. Fractiles are additive under the assumption of perfect positive correlation. Shading indicates not applicable]

\begin{tabular}{|c|c|c|c|c|c|c|c|c|c|c|c|c|c|c|}
\hline \multirow{3}{*}{$\begin{array}{l}\text { Total petroleum systems } \\
\text { (TPS) } \\
\text { and assessment units (AU) }\end{array}$} & \multirow{3}{*}{$\begin{array}{l}\text { Field } \\
\text { type }\end{array}$} & \multirow{3}{*}{$\begin{array}{l}\text { Expected } \\
\text { largest } \\
\text { field size }\end{array}$} & \multicolumn{12}{|c|}{ Total undiscovered resources } \\
\hline & & & \multicolumn{4}{|c|}{ Oil (MMBO) } & \multicolumn{4}{|c|}{ Gas (BCFG) } & \multicolumn{4}{|c|}{ NGL (MMBNGL) } \\
\hline & & & F95 & F50 & F5 & Mean & F95 & F50 & F5 & Mean & F95 & F50 & F5 & Mean \\
\hline \multicolumn{15}{|c|}{ Nile Delta Basin Province, Mesozoic-Cenozoic Composite TPS } \\
\hline \multirow{2}{*}{ Nile Margin Reservoirs AU } & Oil & 214 & 449 & 1,150 & 2,600 & 1,288 & 551 & 1,425 & 3,237 & 1,597 & 11 & 29 & 66 & 32 \\
\hline & Gas & 766 & & & & & 698 & 2,193 & 5,750 & 2,574 & 21 & 68 & 181 & 80 \\
\hline \multirow{2}{*}{ Nile Cone AU } & Oil & 311 & 42 & 256 & 1,666 & 475 & 152 & 930 & 6,123 & 1,758 & 6 & 38 & 255 & 73 \\
\hline & Gas & 17,194 & & & & & 91,213 & 197,850 & 410,825 & 217,313 & 2,413 & 5,269 & 10,962 & 5,789 \\
\hline \multicolumn{15}{|c|}{ Sirte Basin Province, Sirte-Rachmat Composite TPS } \\
\hline \multirow{2}{*}{ Onshore Sirte Carbonate-Clastic AU } & Oil & 432 & 364 & 1,087 & 2,823 & 1,278 & 418 & 1,338 & 4,035 & 1,673 & 22 & 74 & 240 & 96 \\
\hline & Gas & 2,042 & & & & & 1,267 & 4,179 & 12,569 & 5,169 & 44 & 151 & 478 & 192 \\
\hline \multirow{2}{*}{ Offshore Sirte Basin AU } & Oil & 857 & 563 & 1,838 & 5,457 & 2,267 & 633 & 2,250 & 7,677 & 2,972 & 34 & 124 & 454 & 170 \\
\hline & Gas & 6,843 & & & & & 6,591 & 19,540 & 49,077 & 22,637 & 233 & 709 & 1,903 & 840 \\
\hline \multicolumn{15}{|c|}{ Pelagian Basin Province, Bou Dabbous-Cenozoic TPS } \\
\hline \multirow{2}{*}{$\begin{array}{l}\text { Bou Dabbous-Cenozoic } \\
\text { Structural/Stratigraphic AU }\end{array}$} & Oil & 60 & 130 & 283 & 552 & 305 & 45 & 113 & 274 & 131 & 1 & 3 & 8 & 4 \\
\hline & Gas & 616 & & & & & 1,443 & 2,933 & 5,405 & 3,119 & 37 & 75 & 140 & 80 \\
\hline \multicolumn{15}{|c|}{ Pelagian Basin Province, Jurassic-Cretaceous Composite TPS } \\
\hline \multirow{2}{*}{$\begin{array}{l}\text { Jurassic-Cretaceous } \\
\text { Structural/Stratigraphic AU }\end{array}$} & Oil & 13 & 62 & 116 & 212 & 124 & 79 & 154 & 287 & 165 & 2 & 4 & 7 & 4 \\
\hline & Gas & 569 & & & & & 1,044 & 2,409 & 5,023 & 2,643 & 32 & 73 & 154 & 80 \\
\hline & & & Trias & Ghadame & Basin Pro & vince, $\mathrm{Pal}$ & zzoic Comp & site TPS & & & & & & \\
\hline Oued Mya Paleozoic & Oil & 96 & 631 & 1,004 & 1,539 & 1,035 & 480 & 768 & 1,180 & 791 & 20 & 32 & 49 & 33 \\
\hline Reservoirs AU & Gas & 249 & & & & & 363 & 753 & 1,573 & 831 & 24 & 54 & 128 & 63 \\
\hline Melrhir Paleozoic & Oil & 12 & 195 & 287 & 419 & 294 & 103 & 153 & 225 & 157 & 4 & 6 & 9 & 6 \\
\hline Reservoirs AU & Gas & 65 & & & & & 571 & 861 & 1,270 & 883 & 52 & 79 & 117 & 81 \\
\hline Berkine Paleozoic and & Oil & 194 & 1,101 & 1,786 & 2,777 & 1,839 & 2,484 & 4,169 & 6,792 & 4,338 & 130 & 221 & 365 & 231 \\
\hline Mesozoic Reservoirs AU & Gas & 450 & & & & & 1,157 & 2,138 & 3,751 & 2,255 & 72 & 135 & 244 & 143 \\
\hline & & Hamra & asin Pro & nce, Silu & n/Upper D & evonian- & nadames/B & rkine Com & site TPS & & & & & \\
\hline Hamra Deep Structures & Oil & 69 & 124 & 430 & 841 & 451 & 232 & 836 & 1,801 & 902 & 9 & 32 & 76 & 36 \\
\hline and Subcrop AU & Gas & 378 & & & & & 556 & 2,010 & 4,088 & 2,130 & 51 & 195 & 437 & 213 \\
\hline Hamra Basin Margin & Oil & 73 & 42 & 190 & 499 & 220 & 79 & 369 & 1,042 & 439 & 3 & 14 & 43 & 18 \\
\hline Structures AU & Gas & 266 & & & & & 79 & 400 & 1,411 & 525 & 8 & 39 & 143 & 52 \\
\hline Al A tshan Saddle AU & Oil & 44 & 13 & 66 & 232 & 87 & 25 & 130 & 476 & 174 & 1 & 5 & 20 & 7 \\
\hline 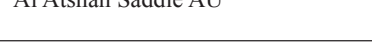 & Gas & 267 & & & & & 81 & 399 & 1,421 & 526 & 7 & 39 & 145 & 53 \\
\hline Sinto Doin Cuborat & Oil & 19 & 0 & 18 & 86 & 26 & 0 & 35 & 175 & 52 & 0 & 1 & 7 & 2 \\
\hline Silte Dasilt Suociop AU & Gas & & & & & & 0 & 0 & 0 & 0 & 0 & 0 & 0 & 0 \\
\hline & & & & Illizi Basi & Province, & Paleozoic & omposite 1 & & & & & & & \\
\hline Jllizi Paleozoic Reservoirs AU & Oil & 177 & 537 & 973 & 1,650 & 1,017 & 1,998 & 3,673 & 6,456 & 3,884 & 35 & 65 & 115 & 69 \\
\hline 1 Ilizi Paleozoic Reservoirs AU & Gas & 1,411 & & & & & 7,124 & 11,800 & 18,515 & 12,176 & 508 & 879 & 1,458 & 918 \\
\hline & & & Gran & Erg/Ahn & Basin Pro & vince, $\mathrm{Pal}$ & ozoic Comp & site TPS & & & & & & \\
\hline Gourara Paleozoic Reservoirs AU & Oil & 22 & 125 & 217 & 365 & 227 & 275 & 493 & 846 & 518 & 5 & 9 & 15 & 9 \\
\hline 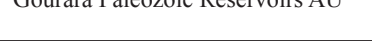 & Gas & 1,125 & & & & & 8,538 & 14,893 & 24,989 & 15,559 & 14 & 25 & 47 & 27 \\
\hline Ahnet Paleozoic & Oil & & 0 & 0 & 0 & 0 & 0 & 0 & 0 & 0 & 0 & 0 & 0 & 0 \\
\hline Reservoirs AU & Gas & 155 & & & & & 2,419 & 3,994 & 6,571 & 4,176 & 3 & 4 & 7 & 4 \\
\hline & & & Essaol & ra Basin & vince, $\mathrm{Pa}$ & leozoic-N & sozoic Con & posite TPS & & & & & & \\
\hline Fssaouira Basin $\mathrm{AU}$ & Oil & 42 & 52 & 116 & 246 & 128 & 53 & 122 & 268 & 136 & 1 & 3 & 7 & 4 \\
\hline Essaounta Dasill AU & Gas & 253 & & & & & 315 & 691 & 1,474 & 766 & 10 & 22 & 49 & 25 \\
\hline Offechere Solt Structures $A \mathrm{U}$ & Oil & 1,788 & 2,416 & 6,715 & 15,496 & 7,557 & 3,794 & 10,759 & 25,476 & 12,198 & 100 & 286 & 697 & 328 \\
\hline Unsnore Salt Suructures AU & Gas & 10,778 & & & & & 14,681 & 40,352 & 92,348 & 45,208 & 896 & 2,482 & 5,759 & 2,791 \\
\hline $\begin{array}{l}\text { Total conventional } \\
\text { resources }\end{array}$ & & & 6,846 & 16,532 & 37,460 & 18,618 & 149,541 & 335,112 & 712,430 & 370,375 & 4,809 & 11,244 & 24,785 & 12,553 \\
\hline
\end{tabular}




\section{References Cited}

Kirschbaum, M.A., Schenk, C.J., Charpentier, R.R., Klett, T.R., Brownfield, M.E., Pitman, J.K., Cook, T.A., and Tennyson, M.E., 2010, Assessment of undiscovered oil and gas resources of the Nile Delta Province, Eastern Mediterranean: U.S. Geological Survey Fact Sheet FS 2010-3027, 4 p.

U.S. Geological Survey World Energy Assessment Team, 2000, U.S. Geological Survey World Petroleum Assessment 2000Description and results: U.S. Geological Survey Digital Data Series DDS-60, 4 CD-ROMs.

Whidden, K.J., Lewan, M.D., Schenk, C.J., Charpentier, R.R., Cook, T.A., Klett, T.R., and Pitman, J.K., 2011, Assessment of undiscovered oil and gas resources of Libya and Tunisia, 2010: U.S. Geological Survey Fact Sheet FS 2011-3105, 2 p.

\section{For Further Information}

Supporting studies of the geologic models and the methodology used in the assessment of North African provinces are in progress. Assessment results are available at the USGS Energy Program Web site, http://energy.cr.usgs.gov/oilgas/.

\section{North Africa Provinces Assessment Team:}

Christopher J. Schenk, Timothy R. Klett, Katherine J. Whidden, Mark A. Kirschbaum, Ronald R. Charpentier, Troy A. Cook, Michael E. Brownfield, and Janet K. Pitman.

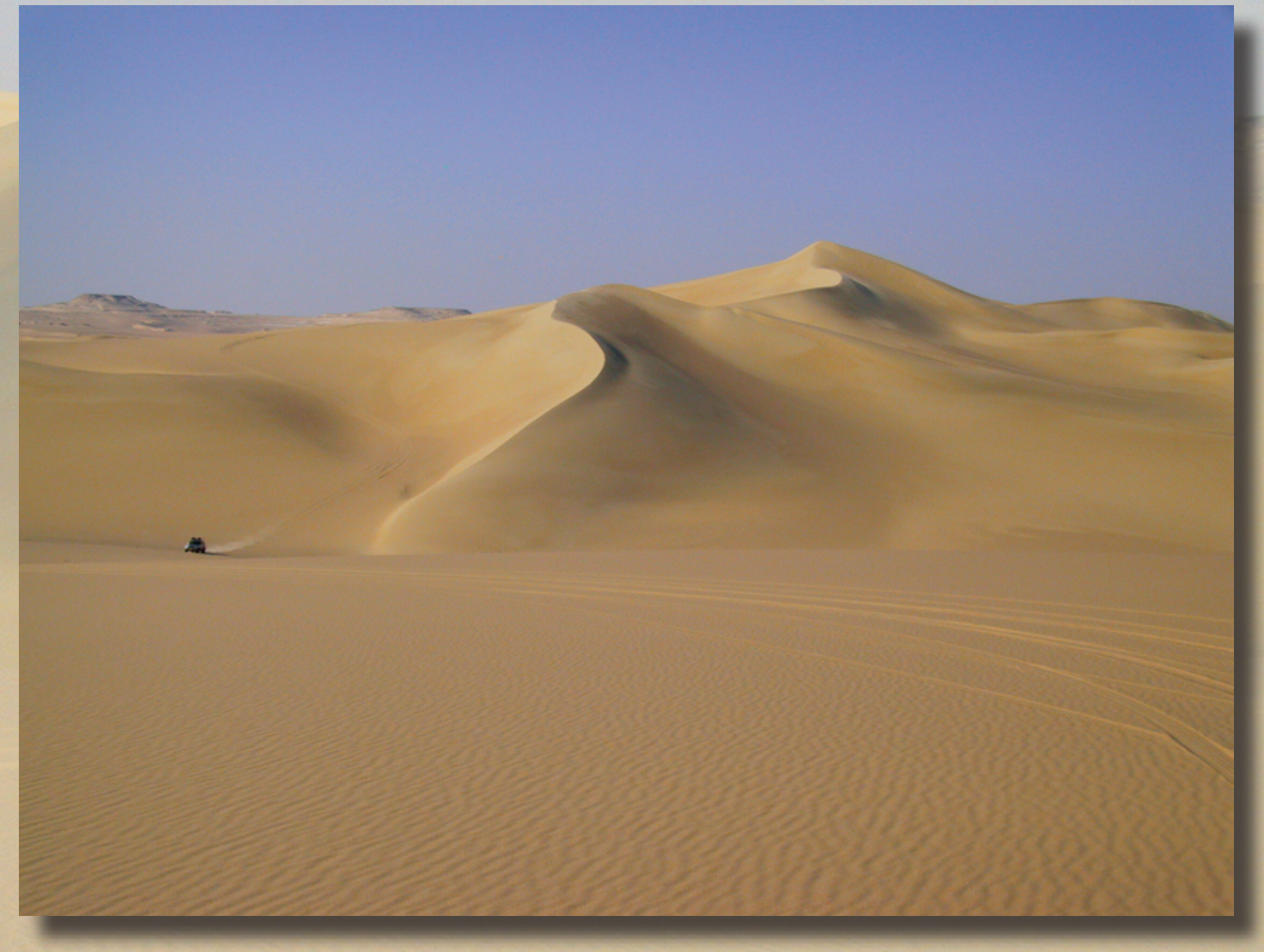

Sinuosity in a linear dune, Sahara Desert, Egypt. Note four-wheel drive vehicle for scale. Photo by K.J. Whidden, 2005. 